\title{
Violencia urbana: análisis crítico y limitaciones del concepto
}

\author{
Sebastían Saborío
}

Artículo

Invitado nacional

Institución: Escuela de Sociología de la Universidad de Costa Rica

E-mail: sebastian.saborio@gmail.com

Recibido: 3 de mayo de 2018

Aprobado: 6 de diciembre de 2018

\section{Sebastían Saborío}

Licenciado en Ciencias Políticas y Relaciones Internacionales y Master en Sociología por la Universidad de Padua, Italia. Doctor en Sociología por la Universidade Federal do Rio de Janeiro y por la Università degli Studi di Urbino. Docente de la Escuela de Sociología de la Universidad de Costa Rica.

\section{Resumen:}

Hasta el momento no existe una definición de violencia urbana que sea considerada como válida por la comunidad académica. Sin embargo, esto no impide que en las ciencias sociales el concepto se empleé con regularidad. En la mayoría de los casos, las publicaciones científicas lo utilizan de manera acrítica como sinónimo de crimen y homicidios. En otros, se proponen definiciones que se limitan a describir las características de la violencia en contextos urbanos específicos y que no pueden ser generalizadas a otras realidades sociales. A través de los resultados de un análisis bibliográfico, el autor explica la importancia de verificar la validez del concepto y expone cuáles son sus principales limitaciones y debilidades. Cuando se refieren a la violencia urbana, las ciencias sociales suelen tomar en consideración principalmente aquellas formas de violencia altamente visibles y que consiguen hacer que una ciudad sea identificada como violenta, disminuyendo la percepción de seguridad de las personas. El principal problema del concepto radica en el hecho de que éste se emplea sobre todo para describir la violencia que ocurre en espacios donde usualmente viven poblaciones socialmente excluidas, las cuales no tienen el poder de incidir en su definición y uso. En consecuencia, el uso acrítico puede consolidar representaciones que estigmatizan y criminalizan a tales poblaciones y los lugares en donde viven; $y$, a su vez, refuerzan potencialmente los discursos y políticas de seguridad de matriz punitiva y excluyente.

Palabras Clave: limitaciones; violencia urbana; visibilidad.

\section{Urban Violence: critical analysis and limitations of the concept}

Keywords: limitations; urban violence; visibility. 


\section{Introducción}

n las ciencias sociales no existe una definición de "violencia urbana" que sea aceptada como válida y que dé cuenta de su especificidad dentro del espectro de las diversas manifestaciones de la violencia. Body-Gendrot (1995) fue la primera en notar tal ausencia e instar a la comunidad académica a definir claramente el concepto. Moser y Mcllwaine (2004) hacen hincapié sobre el hecho de que la violencia urbana es actualmente medida y clasificada de diferentes maneras. En una publicación más reciente, Salahub, Gottsbacher y de Boer (2018) afirman que, según el lugar donde se lleve a cabo y la persona que lo analice, el concepto de violencia urbana puede tener diferentes significados. El presente artículo no tiene el objetivo de avanzar hacia la elaboración de una definición del concepto. Por el contrario, aquí se delineará una crítica al uso que se hace del mismo en las publicaciones académicas basadas en sus propias limitaciones: la heterogeneidad de manifestaciones empíricas a las cuales se refiere (actos y prácticas violentas) y la reproducción de representaciones sociales dominantes hacia poblaciones marginalizadas que puede alimentar y justificar retóricas punitivas.

El dilema sobre la definición del concepto de violencia urbana se remonta, en parte, a las múltiples definiciones y pluralidad de significados que tiene el mismo. Según Imbusch (2005), la violencia se puede dividir en: abstracta, directa, institucional, estructural y simbólica. Como hace notar Calderón "el concepto ha pasado a significar casi cualquier cosa" (2018, p. 101). No cabe aquí ahondar en cada uno de los significados que han sido atribuidos a la violencia, sobre todo porque en la producción académica el concepto de violencia urbana se emplea principalmente como violencia directa. Esto significa que se implementa el concepto de la manera que Calderón considera ser su sentido original, es decir "la amenaza o uso de la fuerza para alcanzar un determinado propósito u objetivo" (2018, p. 101).

En el intento de comprender la forma en que se utiliza el concepto desde las ciencias sociales, Carrión (2008, p. 114) nos dice que se tienen tres acepciones diferentes de violencia urbana "una, que le endilga a lo urbano la cualidad causal; otra, que le asigna la condición de un tipo particular de violencia; y la tercera, que la presenta como si fuera símil de violencia". Sin embargo, la aseveración del autor no tiene un respaldo suficiente de datos que nos muestren que tal división corresponde al uso efectivo del concepto, lo que nos impide aceptarla y nos obliga a someterla a una verificación ulterior, no tanto para definir la violencia urbana, si no para comprender el uso del concepto en las publicaciones científicas.

La mayoría de las publicaciones académicas que tratan el tema de la violencia urbana lo hacen sin explicitar claramente que es lo que entienden por tal. De esta manera, los autores parecen insinuar que, para ser denominada como "urbana", es suficiente que una forma de violencia se lleve a cabo dentro de un contexto urbano. Sin embargo, esto no explicaría por qué cuando se analizan algunas formas de violencia -doméstica, por ejemplo, como veremos más adelante-, estas no se incorporan dentro del debate sobre violencia urbana, aunque se llevan a cabo en las ciudades.

En otros casos, en cambio, el concepto viene asociado, de manera más o menos explícita, a la tasa de homicidios y de otras formas de criminalidad presentes dentro de una ciudad. Por ejemplo, dentro de 76 publicaciones científicas en inglés, español y portugués que analizan principalmente el contexto latinoamericano y, en las cuales, se encuentra el término violencia urbana, sólo 5 autores (9 publicaciones en total ${ }^{2}$ ) brindan una definición elaborada de a lo que se refieren con este. Incluso, en 37 de estos trabajos las palabras "violencia urbana" forman parte del título. Ni siquiera los

1 Todas las citas de textos en idiomas diferentes al español han sido traducidas por parte del autor.

2 Machado da Silva (2004; 2008; 2010b; 2011), Misse (2008; 2011), Misse \& Grillo (2014); Burgos (2005); Harroff-Tavel (2012). autores del libro "Violência Urbana" (Pinheiro \& Assis de Almeida, 2003) dan una definición de la misma y en el texto se limitan a mencionar que es la violencia (en un sentido amplio), la violencia interpersonal y la criminalidad urbana. Es claro que las 76 publicaciones tomadas en consideración no conforman la totalidad de las que adoptan el concepto, pero representan una muestra significativa del uso que se hace del mismo en los debates académicos. Además, para llegar a afirmar que no existe una definición de violencia urbana que sea aceptada como válida entre los miembros de la comunidad científica, el presente artículo se basa también en los resultados de una investigación 
de Muggah (2012). Este autor realizó una recolección bibliográfica usando la palabra clave "violencia urbana" en motores de búsqueda académicos, llegando a la conclusión de que las definiciones y caracterizaciones que se hacen de la violencia urbana comúnmente son rechazadas por parte de otros académicos, ya que son consideradas insuficientes de acuerdo con lo mencionado anteriormente, es decir "no existe una definición de que es lo que constituye la violencia urbana” (Muggah, 2012, p. 19).

Algunos de los autores que no brindan una definición específica se limitan a elaborar listados y categorizaciones de formas de violencia que, según los mismos, conforman la violencia urbana. Sin embargo, en estos casos también notamos la ausencia de una explicación de cuáles son los cimientos empíricos y teóricos que llevan a tales autores a delimitar el concepto a determinadas formas específicas de violencia y no a otras. Por ejemplo, en el caso de Burgos (2005, p. 198) no es claro por qué sostiene que la violencia urbana es "el aumento exponencial de homicidios, robos, asaltos a mano armada, guerra entre traficantes de drogas y delincuencia juvenil". De la misma manera, Burke (1999, p. 3) no explica por qué "el homicidio no es la única forma de violencia urbana, [y, para comprenderla] tenemos que estudiar también la violación, la destrucción de propiedad privada y las lesiones corporales".

Otros autores elaboran clasificaciones más complejas de las formas de violencia urbana sin explicar los parámetros que tomaron en cuenta para tal fin y la utilidad que puede tener una subdivisión en categorías de violencia para legitimar el uso del concepto. Entre estas clasificaciones, la que ha tenido una mayor difusión en el debate académico es la de Moser (2004) la cual, en su "road map" de la violencia urbana, propone una subdivisión en cuatro categorías: política, institucional, económica y social.

Para Harroff-Tavel (2012, p. 32) la violencia urbana es, simplemente, el "uso de la fuerza en ambientes urbanos o peri-urbanos que conlleva daños físicos y psicológicos, así como también deprivación material". Además del hecho de incluir la deprivación material, es decir, aquella que Galtung (1969) llama "violencia estructural", la posición de Harroff-Tavel coincide con el enfoque mencionado previamente donde se da por sentado que, si una forma de violencia se lleva a cabo en la ciudad esta es, automáticamente, violencia urbana. Lamentablemente, aunque tal definición tiene el mérito de una comprensión inmediata, no consigue individuar los diferentes matices con los cuales el concepto es usado por el resto de la comunidad académica. No consigue, por ejemplo, explicar por qué, como es subrayado por Mcllwaine (1999), Koonings (2012) y Moser (2004), el término violencia urbana a menudo se sobrepone con el de crimen, término que tiene una naturaleza estrictamente legal; o, por qué cuando se habla de violencia urbana, no existe una visión homogénea y aceptada como válida sobre qué la conforma, permitiendo así que se incluyan en este concepto expresiones de violencia tan diferentes en sus formas, causas y consecuencias.

\section{Necesidad de comprender la validez del concepto}

De las 76 publicaciones tomadas en consideración, sólo Body-Gendrot (1995) señaló la necesidad de elaborar una definición de violencia urbana. Otros autores, por ejemplo, Jütersonke, Muggah y Rodgers (2009), se limitan a constatar la heterogeneidad de formas que la constituyen. Esto parece no representar un obstáculo a aquellos que utilizan el término de manera acrítica. Entre estos, Muggah (2014, p.7), aunque reconoce que no es claro aquello que se entiende por violencia urbana, no tiene problemas en afirmar que América Latina es una de las regiones que "tiene una tasa de violencia urbana entre las más altas del mundo". De la misma manera, ¿es legítimo que Adorno (2002, p. 89) diga que en Brasil "no hay datos nacionales en relación a la delincuencia, crimen y violencia urbana" sin que antes haya dado una definición de la misma? ¿Cómo se puede tener datos o medir tasas de algo que no se tiene claro que es? De igual forma, parece inadecuado que para analizar políticas públicas que consiguen disminuir la violencia urbana, Hoelsher y Nussio $(2015$, p. 2) circunscriban su mirada hacia las "formas letales de violencia social urbana - en específico la violencia entre individuos o pequeños grupos que no tienen una motivación política". Esta afirmación podría tener sentido si por violencia urbana se entendieran los homicidios de manera unánime y excluyente, pero no es así. Sin embargo, son numerosos los estudios que tienen como 
objetivo comprender la violencia urbana y terminan por focalizarse únicamente en los homicidios, sus causas y consecuencias. Queda claro, a partir de lo dicho, que no tiene sentido desarrollar investigaciones policy-oriented que buscan soluciones para la violencia urbana, mientras no se tenga claro de que se está hablado y mientras no exista un consenso al respecto. Es evidente que, desde el momento que la misma se percibe de manera distinta acorde a quien la enuncia, se buscarán causas y soluciones diferentes y se usarán métodos y técnicas de medición diferentes.

En esta perspectiva, por ejemplo, los resultados de investigaciones que analizan la violencia urbana, tomando en consideración el número de heridos que llegan al servicio de Emergencias en un hospital (Shepher et al., 1990; 1993), no pueden servir para analizar la violencia urbana cuando esta se refiere a daños a la propiedad privada (Burgos, 2005). Lo dicho hasta ahora nos muestra la inconsistencia del concepto de violencia urbana, lo que nos da los primeros elementos para interrogarnos sobre la validez de su uso en el debate académico.

\section{Violencia urbana entre representación y realidad}

Para Machado da Silva (2004) la violencia urbana es una representación discursiva que constituye lo que describe y, por tal motivo, no puede ser definida. En otras palabras, para Machado da Silva la violencia urbana es lo que se dice que es y, por ser cambiante y situacional, no tiene sentido definirla. En esta línea se podría decir que dar una definición de violencia urbana equivaldría a reificar una abstracción mental. Para Machado da Silva $(2008$, p. 41) la violencia urbana es una representación discursiva, producto de lo que llama "sociabilidad violenta", que define como la "transformación de la fuerza [que pasa de ser un] medio de obtención de intereses a [un] principio de coordinación de las acciones". Vale decir que, para el sociólogo brasileño, la violencia urbana es el resultado directo de la elevación de la fuerza a un medio regulador de las interacciones de la vida cotidiana.

La fuerza a la que se refiere Machado da Silva es capaz de suspender, pero no de eliminar, el monopolio del Estado sobre la violencia. Al momento de referirse a la violencia urbana, el autor piensa en una forma específica de violencia: la de las bandas de droga que, desde la década de 1980, controlan territorios empobrecidos de las ciudades brasileñas. Machado da Silva se refiere, en particular, a las bandas criminales de Río de Janeiro ${ }^{3}$ las cuales, a través de la imposición de la fuerza, pasaron a sustituir el poder del Estado en las favelas de la ciudad. De hecho, Machado da Silva afirma que "la noción de violencia urbana se restringe casi siempre a la actividad de un tipo de agente específico - el criminal, en particular el traficante de drogas" (p. 20).

Michel Misse (2008) también basa su análisis de la violencia urbana en la ciudad de Río de Janeiro. Para este autor, el surgimiento de la misma se dio a inicios de la década de 1950 , periodo en el que las autoridades pasaron a luchar contra la violencia criminal a través del uso de los "escuadrones de la muerte", produciendo una espiral de violencia que para Misse se define como "acumulación social de la violencia", donde la violencia de los criminales alimenta la del Estado y viceversa. En síntesis, para el sociólogo la violencia urbana existe en las realidades en las que se verifica una acumulación social de la violencia.

Las intuiciones de Machado da Silva y Misse sobre el uso del concepto de violencia urbana, aun cuando sean correctas en relación al contexto brasileño (particularmente al de Río de Janeiro), sirven únicamente para describir los lugares que poseen las mismas características mencionadas anteriormente por los dos sociólogos. En cambio,

3 En este artículo el análisis de la violencia urbana no se delimita a ningún contexto geográfico, sin embargo, la mayoría de la literatura que ha sido tomada en consideración está relacionada a América Latina, en particular a la ciudad brasileña de Río de Janeiro donde el autor llevó a cabo sus estudios doctorales en sociología. a través del análisis de la literatura, vemos que el término se usa también en contextos donde la acumulación social de la violencia y el uso de la fuerza como regulador de la vida cotidiana no se verifican. A este punto es oportuno tomar en consideración la sugerencia de Carrión (2008), que nos incita a analizar la violencia urbana a través de las relaciones sociales existentes en las diferentes ciudades. Esto significa que, para analizar la utilización del concepto, es necesario individuar las características y peculiaridades propias de cada ciudad, lo que nos puede llevar a comprender que lo que se define como violencia urbana depende del contexto en el que se lleva a 
cabo y la percepción que se tiene de la misma. Esto quiere decir que el concepto viene comúnmente usado para referirse a diferentes manifestaciones violentas, lo que nos ayuda a comprender su incapacidad de referirse a un fenómeno unitario y, en consecuencia, la imposibilidad de definirlo como tal.

La violencia urbana tiene que ver más con las representaciones que se hacen de una determinada ciudad que con el nivel de violencia que se presenta. Por esta razón, podemos afirmar que no son sólo las tasas de criminalidad lo que hacen que una ciudad sea identificada como violenta e insegura. Davis (2012) ilustra como una ciudad puede ser pensada de esta manera aun cuando las estadísticas oficiales no lo justifican. Esto significa que el concepto de violencia urbana tiene, antes que todo, un valor discursivo en el cual lo que se dice y se percibe puede tener un peso mayor con respecto a lo que sucede. Según la autora, un buen ejemplo de lo afirmado arriba es Ciudad de México, que normalmente es percibida tan violenta como Medellín, aunque presenta menores niveles de violencia:

La percepción pública se basa en pocos accidentes de alto perfil ${ }^{4}$ en algunos lugares (en particular a lo largo de la frontera, pero también en la capital) que llevaron muchas personas a identificar a México como extremamente violenta. Sin embargo, los índices [de violencia] en México, como nación, y en la Ciudad de México en particular, no alcanzan la violencia en Colombia en general o en Medellín en particular. (Davis, 2012, p. 27)

Tal distorsión de la realidad a través de las representaciones de la violencia no se limita a los países del Sur Global, lo mismo puede suceder en Europa, donde las tasas de criminalidad son marcadamente más bajas que en América Latina. Wacquant (2009, p. 252) nos recuerda como en el 2000 los medios de comunicación describían a Francia como "infestada por la criminalidad" aunque las estadísticas probaban lo contario. De hecho, la tasa de homicidios no era solo muy baja ${ }^{5}$ (3,6 por 100.000 habitantes), sino que, incluso, había disminuido un punto con respecto a 1990. Además, para Wacquant (2009, p. 251) los asaltos, lejos de suceder a "todos y por todo lado" como se hace creer en los medios de comunicación, cada año afectan alrededor del $2 \%$ de la población y se concentran en las periferias urbanas más empobrecidas. Algo parecido se verifica en Italia donde, de acuerdo con Maneri (2013), la "inseguridad percibida", producto de alarmas mediático-políticas, es mucho mayor que la "inseguridad real".

Body-Gendrot (1995, p. 525) sostiene que la violencia urbana se produce de la "interacción entre las representaciones y la realidad que las personas viven en determinados ambientes urbanos". Como diría Martín-Barbero (2002), son las palabras y el uso que se hace de estas para describir los espacios públicos de una ciudad que ayudan a formar la percepción que las personas tienen de la misma. En consecuencia, si entre las palabras que se usan para describir una ciudad, la presencia de imágenes y los discursos relacionados a la violencia se presentan de manera sustancial y constante, esta pasará a ser percibida como violenta. Podemos ver, entonces, que el uso del concepto de violencia urbana suele estar acompañado por representaciones que muestran una determinada ciudad como violenta.

Como es mencionado antes, el concepto de violencia urbana engloba dentro de sí un delicado equilibrio entre representaciones y realidad. Misse y Grillo (2014, p. 55) indican que la percepción de seguridad de las personas depende de una mezcla entre la violencia efectiva que se produce en una determinada ciudad y la representación mediática de la misma:

La experiencia de las calles, de los turistas, de las rupturas de la cotidianidad de sus habitantes por los pequeños y grandes delitos, así como por la cantidad de eventos e historias divulgadas en la prensa, todo eso converge para constituir diferentes signos de "visibilidad". un "peligro social".

De hecho, cuando la experiencia cotidiana que las personas tienen del contexto urbano está plasmada por eventos violentos, la imagen -entendida como "la suma de convicciones, ideales e impresiones que la gente tiene en respeto a un lugar" (Moncada, 2013, p. 314)- será, inevitablemente, la de una ciudad donde el nivel de violencia es elevado. 


\section{Visibilidad de la violencia y percepción de la seguridad}

¿Cuáles son las formas de violencia que producen la imagen de una ciudad como violenta? Para Misse (2011, p. 11) la violencia urbana estárelacionada a una "multiplicidad de eventos" que son propios de la vida urbana moderna y que se relacionan a formas de violencia que son más o menos evidentes. Según Machado da Silva (2011, p. 70), las prácticas que suelen ser relacionadas al concepto van desde la "simple incivilidad hasta la extrema crueldad" $y$, de todos modos, pueden variar de forma con base en la localidad donde se llevan a cabo. El mismo autor (2004, p. 57) agrega que para que estas formas de violencia contribuyan a la representación de la violencia urbana, estas tienen que ser "consideradas como una amenaza a las dos condiciones básicas del sentimiento de seguridad existencial que usualmente acompaña la vida cotidiana - integridad física y garantía de los bienes patrimoniales". Dicho con otras palabras, aquellas formas de violencia que resquebrajan el sentimiento de seguridad de las personas. Esta perspectiva se refleja, por ejemplo, en investigaciones como la de Carvajal y Rivera (2001), donde el análisis de la violencia urbana de la ciudad de San José de Costa Rica se limita a la percepción de los residentes sobre la inseguridad.

Koonings (2012, p. 15) dice que la violencia urbana es "altamente visible" y es por este motivo que puede aumentar la percepción de inseguridad y el miedo entre la ciudadanía. En realidad, no es correcto decir que la violencia urbana es altamente visible, sino que lo son las formas de violencia que normalmente se identifican con tal concepto. Es decir, sostener que la violencia urbana sea altamente visible equivaldría a reificar la misma; mientras que evidenciar las practicas que la componen son visibles, representa un movimiento analítico orientado a especificar tales prácticas. Esto nos permite sostener que las formas de violencia que caracterizan una determinada ciudad como violenta y que, en consecuencia, son asociadas al concepto de violencia urbana son, sobre todo, aquellas altamente visibles. Entre más alto sea el número de episodios de violencia visible que se verifican en una ciudad, mayores posibilidades hay de que esta sea identificada como violenta y que el concepto de violencia urbana sea usado para describirla.

Como demostración del hecho de que las formas de violencia que se incluyen dentro del concepto de violencia urbana son, sobre todo, las más visibles, es importante recordar que muchas veces el concepto es usado simplemente como sinónimo de tasa de homicidios. Estos, efectivamente, gozan de gran visibilidad en los medios de comunicación y consiguen direccionar el debate público y moldear las políticas públicas en relación a la seguridad ciudadana. En cambio, a diferencia de los homicidios, la violencia de género, por llevarse a cabo principalmente dentro de los hogares, posee una menor visibilidad ${ }^{6}$. De hecho, ésta nunca viene tomada en consideración en el debate académico sobre la violencia urbana (Wilding, 2010) aunque, a nivel global, sí es una de las formas de violencia más difundidas (Marc \& Willman, 2011).

Básicamente, la violencia de género, en particular la violencia doméstica, no viene considerada en el debate sobre la violencia urbana por la invisibilización social que sufre el fenómeno. La visibilidad de la violencia depende, en gran medida, del espacio que la misma recibe en los medios de comunicación y, en general, en el debate público. En el contexto de Río de Janeiro en Brasil, Ramos y Paiva (2007) evidencian el hecho de que, aunque las violaciones son uno de los principales problemas de seguridad que tiene la ciudad, estas reciben poca atención en los periódicos locales. Por ejemplo, en el 2006 se publicaron 593 noticias sobre hechos violentos y de estas sólo una estaba relacionada a la violencia de género. La mayoría de estas noticias narraban conflictos armados entre bandas criminales y robos.

Para que una acción violenta sea visible socialmente, la naturaleza de la misma es tan importante como el lugar en donde se lleva a cabo, las personas que victimiza y, sobre todo, el sujeto que la lleva a cabo. Como se mencionó, crimen y violencia son dos conceptos separados, sin embargo, el análisis de la criminalidad nos permite comprender mejor la distribución de la visibilidad social entre las diferentes formas de violencia. La sociología del derecho italiana ha demostrado ampliamente que, entre las diferentes formas de criminalidad, la que se verifica en las calles de las ciudades genera un nivel mayor de inseguridad en las personas. Esto no significa que la criminalidad 
callejera sea la más peligrosa o que, en general, produzca mayores daños a la sociedad, sino que para Baratta (2001) la criminalidad de las élites, como por ejemplo los delitos económicos, ambientales, la corrupción, es subrepresentada en el debate público e ignorada por parte de las políticas de seguridad. Sin embargo, este tipo de criminalidad crea enormes daños sociales ya que "produce pobreza, dispersión de los recursos públicos, aumento de los accidentes laborales, enfermedades profesionales y contaminación, trabajo infantil y disgregación social" (Baratta, 2001, p. 34).

Melossi (2002) sostiene que la criminalidad de las clases excluidas provoca una mayor alarma y escándalo social, porque la gran cantidad de los delitos en que estas incurren son muy visibles. Dicho de manera distinta, la criminalidad es más aceptada y normalizada cuanto más se ejerce "sin provocar un escándalo público" (Melossi, 2002, p. 277).

\section{Violencia y poder de definición}

Si sustituimos el concepto de criminalidad con el de violencia nos daremos cuenta de que el resultado no cambia. Cuando a ser identificadas como responsables de acciones violentas o criminales son las categorías sociales expoliadas de sus derechos fundamentales, la visibilidad criminalizante de sus acciones es mucho mayor. Esto porque "violencia y poder están inextricablemente correlacionados" (Moser \& Mcllwaine 2004 , p. 9). El concepto de violencia urbana, asociado como normalmente se hace a la violencia que mayormente se visibiliza - la de los pobres y excluidos-, no puede ser considerado un concepto política o científicamente neutro, ya que reproduce, a su vez, las desigualdades sociales existentes.

Si bien sólo algunas formas de violencia pasan a ser consideradas como violencia urbana, es decir, visible y capaz de disminuir la percepción de seguridad de la ciudadanía, es necesario comprender quién tiene el poder de decidir cuales formas de violencia poseen, o no, tales características.

Como nos recuerda Waddington (1999, p. 96) "los sujetos que mantienen posiciones de poder tienen una influencia mayor en definir que es o no es aceptable". En general, lo que percibimos como violencia puede ser considerado el resultado de una construcción social, donde quien posee más recursos puede "establecer o definir no solo qué es violento y qué no, pero también bajo qué circunstancias una acción violenta es legítima o no" (Calderón, 2018, p. 101).

No todas las personas tienen una visión idéntica sobre cuáles son los grupos sociales peligrosos (Pain, 2001). Sin embargo, son los actos criminales, violentos o no, realizados por parte de las clases sociales más desventajadas que provocan una percepción de inseguridad mayor (Misse, 2010). A lo interno de la gramática de la violencia urbana, los sujetos que poseen menos poder en definirla son aquellos que tienen una mayor posibilidad de ser representados como los perpetradores de la misma. No es casualidad que Feltran (2014) diga que la violencia urbana se constituye a través de asociaciones arbitrarias que tienden a representar los lugares de la pobreza como los espacios, por excelencia, de la violencia urbana, y sus habitantes como los protagonistas de la misma. En el caso brasileño, por ejemplo, las personas que viven en las favelas son, comúnmente, refigurados como los responsables de la violencia urbana porque no tienen el poder de definirla. Por esta razón, como diría Feltran (2014), la violencia urbana no es una categoría nativa de los lugares y de los actores a los cuales es asociada. Vemos como las favelas no sólo son territorios urbanos excluidos socialmente porque sus residentes no tienen pleno acceso a los recursos públicos, sino también porque son excluidos del proceso de definición social de los conceptos que representan la realidad en la que viven.

Las representaciones sociales relacionadas a la violencia urbana dependen también de aquellas que son dominantes en la sociedad y que permiten que las características, verdaderas o imaginadas, de los diferentes grupos sociales sean más o menos visibles. Para Brighenti $(2007 ; 2008 ; 2010)$ la visibilidad intrínseca de los individuos puede generar una plataforma de reconocimiento social cuando favorece la autodeterminación y la autodefinición de los diferentes grupos sociales. Al contrario, para el mismo autor, 
la visibilidad puede ser también un medio de dominación cuando sirve para crear representaciones negativas o que pueden inferiorizar sujetos o categorías sociales. En el segundo caso, las "distorsiones de la visibilidad aumentan distorsiones en las representaciones sociales" (Brighenti, 2008, p. 9). En este sentido, por ejemplo, la hiper-visibilidad de los grupos marginalizados puede ser considerada un instrumento de dominación que se produce y reproduce a través del uso acrítico del concepto de violencia urbana.

En los estudios relacionados al control social, ha sido abundantemente debatido el papel de los medios de comunicación en difundir representaciones negativas de las categorías sociales empobrecidas que, en consecuencia, refuerzan estereotipos y procesos de criminalización. Tales procesos legitiman y estimulan las instancias punitivas que favorecen la creación de políticas represivas y que, a su vez, multiplica la violencia institucional que se lleva a cabo a través de las fuerzas de policía y otras instituciones de control como, por ejemplo, el sistema penitenciario. Por ejemplo, Wacquant (2009, p. 2) nos muestra como conceptos y expresiones tales como "violencia urbana" y "guerra a la criminalidad" han sido utilizados en los medios de comunicación en Estados Unidos y Francia a partir de los años de 1970, con el fin de estigmatizar "jóvenes provenientes de los barrios en declino de la clase trabajadora, desempleados, indigentes, mendigos, drogadictos, prostitutas y migrantes". Esta estigmatización ha sido funcional para la legitimación del desmantelamiento del estado social y su substitución con lo que el autor, llama el "estado penal", es decir, la expansión del aparato penal, en particular del sistema penitenciario, pese al hecho que los índices de criminalidad estuviesen estables 0 , incluso, disminuyendo.

Para algunos, la propaganda, es decir, "la sistemática y estratégica manipulación de las visibilidades narrativas, mitos, representaciones y hechos" (Brighenti, 2010, p. 176) es uno de los principales factores que favorecen los procesos de estigmatización arriba mencionados. Otros, en cambio, sostienen que más que por razones de propaganda, los medios de comunicación de masa brindan una representación estereotipada de los grupos posicionados en los niveles más bajos de la sociedad por razones económicas, de espectacularización y venta de sus productos (Stella, 2008). En la primera perspectiva los pobres son criminalizados por razones de propaganda, sobre todo política. En la segunda por motivos económicos, de audience y para contentar el público consumidor.

\section{Conclusiones}

El artículo demuestra la necesidad de analizar en profundidad el concepto de violencia urbana, su uso y validez. Se explica, por ejemplo, que no se puede medir la violencia urbana o buscar soluciones a la misma, ya que no existe una definición de esta que sea aceptada como válida, y que permita identificar claramente que expresiones de violencia la conforman. En lugar de referirnos a la violencia urbana como si fuese un fenómeno homogéneo y claramente identificable, tenemos que tomar en consideración que el concepto se usa en relación a una multiplicidad de hechos, eventos y situaciones muy diferentes entre ellos. Como consecuencia, lo que normalmente es identificado como violencia urbana depende sobre todo del contexto que se analiza.

En las ciencias sociales, el concepto se refiere, principalmente, a formas de violencia directa y altamente visible que afectan negativamente la percepción de seguridad de las personas, y que permiten que una determinada ciudad sea percibida como violenta. Esto significa que no existen elementos que sean útiles para definir la violencia urbana como un fenómeno unitario. En efecto, dependiendo del contexto analizado, pueden ser diferentes (o ninguna) las formas de violencia que tienen la capacidad de definir una determinada ciudad como peligrosa e insegura.

En otras palabras, el uso del concepto está estrechamente relacionado a la percepción de la ciudad, la cual depende de las representaciones e imaginarios que se producen de la misma y de la violencia que en ella acontece. Sin embargo, la relación entre representaciones y realidad no es lineal y directamente proporcional. Esto significa que una ciudad puede ser percibida como peligrosa y ser analizada a través del concepto de violencia urbana, aun cuando la realidad social a la cual se refieren no sea, de hecho, caracterizada por altos índices de violencia. 
Cabe interrogarse quién tiene la capacidad de definir cuáles son las violencias que determinan la representación de una ciudad en el imaginario colectivo. ¿Para quién tienen que ser consideradas peligrosas?, ¿En qué lugares tienen que llevarse a cabo?, ¿Quiénes tienen que ser las víctimas y los victimarios?

Las relaciones de poder existentes en la sociedad permiten que seala violencia perpetrada por parte de los grupos empobrecidos y desempoderados, al gozar de mayor visibilidad en los medios de comunicación y en el debate público en general; mientras que la violencia y criminalidad de los grupos dominantes viene sistemáticamente invisibilizada socialmente. Por esta razón, la violencia que sucede en los espacios públicos es más visible que la que se verifica en la intimidad del hogar, principalmente de hombres contra mujeres. De la misma manera, la violencia de las clases socioeconómicas más bajas produce un mayor escándalo en el debate público que la de los potentes. En relación a la localidad, la violencia que se lleva a cabo en las zonas de residencia y tránsito de las clases medias y altas y que las victimiza, tiene una mayor visibilidad que la que sucede en los barrios urbano-marginalizados, a no ser que esta incida negativamente en la percepción de seguridad de los primeros. En esta perspectiva, es fácil comprender por qué los homicidios, aunque sean cometidos en territorios caracterizados por elevados niveles de exclusión social, vienen normalmente identificados como la apoteosis de la violencia urbana: son altamente visibles y producen un sentimiento de inseguridad en la totalidad de la población y no sólo en las víctimas directas.

El uso acrítico de un concepto que tiene sus raíces en las asimetrías de poder existentes en las relaciones sociales no puede hacer más que reproducirlas. Por tanto, es momento de dejarlo de lado y comenzar a analizar las formas de violencia específicas sin focalizarnos principalmente en el nivel de visibilidad de las mismas, consiguiendo de esta manera individuar sus causas y consecuencias. Asimismo, las recomendaciones que se propongan en relación a las diferentes formas de violencia corren menos riesgos de producir "políticas de visibilidad", es decir, políticas que miran más a reducir la visibilidad de los problemas sociales que a encontrar soluciones a los mismos. En fin, abandonando el concepto se contribuirá a debilitar la retórica criminalizante y excluyente que identifica las categorías sociales más desaventajadas como las responsables de la "violencia urbana".

\section{Referencias Bibliográficas}

Adorno, S. (2002). Exclusão socioeconômica e violência urbana. Sociologias, 4(8), pp. 84-135.

Baratta, A. (2001). Diritto alla sicurezza o sicurezza dei diritti? En S. Anastasi \& M. Palma (Eds.), La bilancia e la misura. Giustizia, sicurezza, riforme (pp. 19-36). Milan: Franco Angeli.

Barbero, J. M. (2002). The city: between fear and the media. En S. Rotker \& K. Goldman (Eds.), Citizens of fear. Urban violence in Latin America (pp. 25-36). New Brunswick: Rutgers University Press.

Body-Gendrot, S. (1995). Urban violence. A quest for meaning. Journal of Ethnic and Migration Studies, 21(4), pp. 525-536.

Brighenti, A. (2007). Visibility. A Category for the Social Sciences. Current Sociology, 55(3), pp. 323-342.

Brighenti, A. (2008). Visual, Visible, Ethnographic. Etnografia e Ricerca Qualitativa, 1, pp. 2-19.

Brighenti, A. (2010). Visibility in social theory and social research. New York: Palgrave Macmillan.

Burgos, M. B. (2005). Cidade, territorios cidadania. Dados, 48(1), pp. 189-222.

Burke, P. (1999). O "processo civilizador" vencerá o poder das novas armas? Violência urbana e civilização. Publicações-Braudel Papers, 12, pp. 3-14.

Calderón Umaña, R. (2018). Violence and social exclusion in urban contexts in Central America. En J. Erin Salahub, M. Gottsbacher, \& J. de Boer (Eds.), Social 
theories of urban violence in the Global South. Toward safe and inclusive cities (pp. 99 120). New York: Routledge.

Carrión, F. M. (2008). Violencia urbana: Un asunto de ciudad. Eure, 34(103), pp. 111130.

Carvajal, G., \& Rivera, D. (2001). Violencia urbana y criminalidad urbana en la ciudad de San José. Un estudio exploratorio. Diálogos Revista Electrónica de Historia, 2(3), pp. 1-37.

Davis, D. E. (ed.) (2012). Urban resilience in situation of chronic violence. Cambridge: MIT Center for International Studies. https://cis.mit.edu/sites/default/files/ documents/urbanresiliencereport2012.pdf

Erin Salahub, J., Gottsbacher, M., \& de Boer, J. (2018). Social Theories of Urban Violence in the Global South. Toward safe and inclusive cities. New York: Routledge.

Feltran, G. (2014). Crime e periferia. En Raton, J.; Lima, R.; Azevedo, R. Crime, Polícia e Justiça no Brasil (pp. 299-307). São Paulo: Editora Contexto/Fórum Brasileiro de Segurança Pública.

Galtung, J. (1969). Violence, peace, and peace research. Journal of Peace Research, 6(3), pp. 167-191.

Harroff-Tavel, M. (2012). Humanitarian response towards urban violence. En P. Apraxine, A. Duquenne, S. Fetta, \& D. Helly, (Eds.), Urban violence and humanitarian challenges (pp. 32-37). Paris: Institute for Security Studies.

Hoelscher, K., \& Nussio, E. (2015). Understanding unlikely successes in urban violence reduction. Urban Studies, pp. 1-20.

Imbusch, P. (2005). The Concept of Violence. En W. Heitmeyer \& J. Hagan (Eds.), International Handbook of Violence Research (pp. 13-40). New York, Boston, Dordrecht, Londres, Moscú: Kluwer Academic Publishers.

J. P. Shepherd, M A Ai, A O Hughes, B G H Levers (1993) Trends in urban violence: acomparison of accident department and police records. Journal of the Royal Society of Medicine, 86, pp. 87-88.

Jütersonke, O., Muggah, R., \& Rodgers, D. (2009). Gangs, urban violence, and security interventions in Central America. Security Dialogue, 40(4-5), pp. 373397.

Koonings, K. (2012). Definition and trends of urban violence. EnP.Apraxine,A. Duquenne, S. Fetta, \& D. Helly, (Eds.), Urban violence and humanitarian challenges. Paris: Institute for Security Studies, pp. 13-16.

Maneri, M. (2013). Si fa presto a dire «sicurezza». Analisi di un oggetto culturale. Etnografia e Ricerca Qualitativa, 2, pp. 283-309.

Marc, A. Willman, A.M. (Eds.) (2011) Violence in the city. Understanding and Supporting community responses to violence. Washington: The World Bank.

Mcllwaine, C. (1999). Geography and development: violence and crime as development issues. Progress in Human Geography, 23(3), pp. 453-463.

Melossi, D. (2002). Stato, controllo sociale, devianza. Milán: Mondadori.

Misse, M. (2008). Sobre a acumulação social da violência no Rio de Janeiro. Civitas Revista de Ciências Sociais, 8(3), pp. 371-385.

Misse, M. (2010). Crime, sujeito e sujeição criminal. Aspectos de uma contribuição analítica sobre a categoria bandido. Lua Nova, 79, pp. 15-38.

Misse, M. (2011). Crime e violência no Brasil contemporâneo: estudos de sociologia do crime e da violência urbana [2nd edition]. Rio de Janeiro: Lumen Juris.

Misse, M., \& Grillo, C. C. (2014). Río De Janeiro. Sufrir la violencia, decir la paz. In A. M. Jaramillo \& C. M. Perea (Eds.), Ciudades en la encrucijada: violencia y poder criminal en Río de Janeiro, Medellín, Bogotá y Ciudad Juárez (pp. 49-116). Medellín: Corporación Región. 
Moncada, E. (2013). Business and the politics of urban violence in Colombia. Studies in Comparative International Development, 48(3), pp. 308-330.

Moser, C. (2004). Urban violence and insecurity. An introductory roadmap. Environment \& Urbanization, 16(2), pp. 1-16.

Moser, C., \& Mcllwaine, C. (2004). Encounters with violence in Latin America. Urban perceptions from Colombia and Guatemala. Londres: Psychology Press.

Muggah, R. (2012). Researching the urban dilemma: Urbanization, Poverty and Violence. Recuperado dehttp://www.hasow.org/uploads/trabalhos/98/doc/1401774567.pdf

Muggah, R. (2014). Deconstructing the fragile city. Exploring insecurity, violence and resilience. Environment and Urbanization, 26(2), pp. 345-358.

Pain, R. (2001). Gender, race, age and fear in the city. Urban Studies, 38(5-6), pp. 899913.

Pinheiro, P. S. de M. S., \& Almeida, G.A. (2003). Violência urbana (Vol. 57). São Paulo: Publifolha.

Ramos, S., \& Paiva, A. (Eds). (2007). Mídia e Violência: novas tendências na cobertura de criminalidade e segurança no Brasil. Río de Janeiro: CESeC.

Shepherd, J.P., Robinson, L., \& Levers, B.G. (1990). Roots of urban violence. Injury, 21(3), pp.139-141.

Silva, L.A.M. (2004). Sociabilidade violenta: por uma interpretação da criminalidade contemporânea no Brasil urbano. Sociedade e Estado, 19(1), pp. 53-84.

Silva, L.A.M. (2008). Vida sob cerco: violência e rotina nas favelas do Rio de Janeiro. Río de Janeiro: Nova Fronteira

Silva, L.A.M. (2011). Polícia e violência urbana em uma cidade brasileira. Etnográfica, 15(1), pp. 67-82.

Stella, R. (2008). Media ed etica: regole e idee per le comunicazioni di massa. Roma: Donzelli.

Waddington, P. A. (1999). Policing citizens: authority and rights. Londres: Psychology Press.

Wacquant, L. (2009). Punishing the poor- The neoliberal government of social insecurity. Critical Social Policy. Durham, Carolina del Norte, "EUA": Duke University Press.

Wilding, P. (2010). «New Violence». Silencing women's experiences in the favelas of Brazil. Journal of Latin American Studies, 42(4), pp. 719-747.

World Bank. (s.f.). Intentional homicides (per 100,000 people). Recuperado de: https:// data.worldbank.org/indicator/VC.IHR.PSRC.P5 